
\title{
R Reserach S Suare \\ The efficacy of diaphragmatic plication in patients with unilateral diaphragmatic paralysis; a two-centre experience
}

Mohamed Abdel Bary Ibrahim ( $\sim$ Dr_abdelbary@med.svu.edu.eg )

Qena faculty of medicine - south Valley university https://orcid.org/0000-0002-7734-9089

\section{Alaa Rashad}

Qena Faculty of Medicine, South Valley University

Hamed Elgendy

Assiut University Faculty of Medicine

Morris Beshay

Evangelisches Klinikum Bethel gGmbH

Research article

Keywords: Diaphragm Diaphragmatic Eventration Diaphragmatic Eventration/surgery

diaphragm/ultrasonography, fluoroscopy, Recovery of Function Thoracic Surgical Procedures/methods

Posted Date: February 11th, 2020

DOl: https://doi.org/10.21203/rs.2.23144/v1

License: (a) This work is licensed under a Creative Commons Attribution 4.0 International License. Read Full License 


\section{Abstract}

Background and objective: Diaphragm is not an uncommon problem. is the most presenting in most of the cases. In this study, we used diaphragm (DUS) and to evaluate the outcome of post-surgical diaphragmatic plication (SDP) in adults with unilateral diaphragmatic paralysis (UDP). To our knowledge, no large reports about the efficacy of and the utilization of DUS for these patients were done.

Methods: A retrospective analysis of all patients who underwent SDP at two (2014 - 2018) was done. Data of all patients were patients' characteristics, preoperative (chest X-ray, chest computed tomography, DUS, and ) , two years postoperative follow up.

Results: Among 47 (UDP) patients. 40 patients underwent SDP. 30 (75\%) males (mean age $45 \pm 14$ years). Left-sided SDP was found in $57 \%$ of cases $(n=23)$. Most of the patients were suffering from Dyspnea $(n=38)$. Minor complications occurred in $12 \%(n=5)$. Excellent results in $90 \%(n=36)$, good results in $7,5 \%$ and unsatisfactory in one patient (2,5\%). Patients up at one month and 6 months and two years showed a significant increase in FEV1 up to $20 \%$ (range $15-40 \%$ ), $P=0.011$ and $F V C$ up to $30 \%$ (range $10 \% 45 \%$ ) $\mathrm{P}=0.024$. There was a significant postoperative improvement in $(\mathrm{P}<0.005)$. There was a significant correlation between postoperative FEV1 and the height of the copula on CXR and DUS $(P<0.001$ and $\mathrm{P}<0.005$ respectively).

Conclusion: SDP is an effective and safe procedure which can be performed to treat UDP in adult patients suffering from chronic . is a significant improvement in the patient's functional is correlated to the assessment. Keywords: Diaphragm Diaphragmatic Eventration Diaphragmatic Eventration/surgery diaphragm/ultrasonography, fluoroscopy, Recovery of Function Thoracic Surgical Procedures/methods

\section{Introduction:}

Unilateral diaphragmatic paralysis (UDP) is defined as a fixed hemidiaphragm raising without defects in its continuity. It occurred due to phrenic nerve dysfunction. It is a fairly uncommon entity with an incidence of $₫ 0.05 \%$, males are more affected [1,2]. Nerve palsy occurs on both sides with slightly more on the left side. It is usually discovered accidentally during routine chest X-ray (CXR), but orthopnoea and dyspnea are the most frequent complaints. Dyspnea occurred due to compression of the affected lung especially the lower lobe, which would be obvious during sports activities or bowing down. In both cases a paradoxical diaphragm movement occurs which accelerates lower lobe compression, building lung atelectasis with the consequence of disturbance of the gas exchange through shunt effect (Fig. 1) $[3,4]$.

Congenital UDP is a very rare condition, but post cryoablation intervention, malignancy, trauma, infection, cervical spondylosis, post chest or cardiac surgery and neuromuscular disorders are the most common acquired causes of diaphragmatic paralysis (DP) [5].

Most of the patients are presented to their family doctors of pneumologist with shortness of breath. Frequently they are not able to go up a hill, have difficulties to walk upstairs or run. Few patients are 
discovered accidentally, who did not have such severe symptoms. Apart from cryoablation, idiopathic phrenic nerve palsy is the most frequent presentation when no definite aetiology found. No treatment is required for asymptomatic cases. But, in symptomatic DP should be managed by surgical plication [6, 7]. latrogenic UDP due to nerve injury showed be observed for up to 2 years, because it may improve with time. However, in severe cases observation should be limited to 6 months before surgery done $[8,9]$.

Surgical diaphragmatic plication (SDP) has become the rationale in paediatric patients suffering from respiratory failure, but it still rarely done in the adult population. This might be due the reluctant of the family doctors and the pneumologist to refer the patients, the reluctant of the surgeons to operate the patients, the lake of experience to do the procedure especially in minimally invasive technique, and finally the lake of publicity of the SDP $[10,11]$.

SDP is to alleviate the shortness of breath by limitation of diaphragmatic elevation during inspiration, which relief the lung atelectasis on the affected side with correction of the disturbed gas exchange. In this procedure, the eventrated paralysed copula is folded on itself and fixed with nonabsorbable sutures. The diaphragm becomes thereafter taut and comes down to its ordinary position. This prompts symptoms improvement occurs through different mechanisms; lung re-expansion, recruitment of more lung volumes needed for gas exchange, reduction of the diaphragmatic paradoxical movement so that the respiratory mechanics can act successfully, correction of the displaced abdominal organs and finally correction of the shunt effect [12].

Recently, diaphragm ultrasonography (DUS) has been evolved as a simple, cheap, promptly accessible, reproducible and bed-side examination for appraisal of the dynamic movements of the diaphragm and its function. Many researchers reported its value for the anticipation of weaning outcomes in critically ill, mechanically ventilated patients [13].

To our knowledge, no large reports about the efficacy of SDP and the utilization of DUS for the postoperative evaluation of UDP patients were done. In this study, we used DUS and spirometry to evaluate the outcome of SDP procedure in adults suffering from symptomatic UDP.

\section{Methods:}

Patients and sampling procedures:

This study included 40 cases with UDP admitted to both centres during the period of 5 years between January 2014 to December 2018. The data of all patients were collected from hospital patients' records, family doctors and personal patients contact. All patients underwent clinical dyspnea evaluation according to MRC score, CXR, chest computed tomography, DUS and spirometry with the assessment of forced expiratory volume 1 (FEV1), forced vital capacity (FVC), FEV1/FVC, these investigations were done at baseline, one month, 6 months, and two years postoperatively. Under DUS and CXR the level of eventrated diaphragm was determined before surgery. A fixed point on the bony structure which was the uppermost of the upper border of the 1st rib; while the patient is setting. Another measurement was done 
compared with the copula of the healthy side pre and postoperatively. The degree of postoperative change was calculated and compared with the degree of change in CXR. The preoperative values of the spirometry were compared with the postoperative values after 6 months and two years. The study conforms with the ethical standards of the Helsinki Declaration and approval was obtained from the local institutional ethics committee. The requirement for informed consent was waived.

\section{Surgical procedures:}

The plication was done by the two surgeons either via a small incision in the eighth intercostals space or using VATS procedure on the affected side (camera trocar and $5 \mathrm{~cm}$ utility incision) according to the degree of the diaphragmatic eversion. The procedure was performed under double lumen intubation general anaesthesia. A nasogastric tube was inserted in all cases. The markedly raised copula was observed in all cases. The diaphragm with the abdominal contents was pushed caudally until the normal position of the copula achieved. At this position, a fold of about $5 \mathrm{~cm}$ is made using traction forceps of the reluctant diaphragm (Fig 2). Polypropylene sutures were used to perform the plication of the diaphragm through making a fold on itself without tissue resection. The plication was performed using interrupted polypropylene U-stitches; usually, it started from the posterior part to the anterior part of the diaphragm (Fig. 3). The diaphragm now becomes tough and firm and returned to its normal position. Caution is to be taken to avoid overcorrection of the diaphragm, as it might have much pressure on the other side of the diaphragm or it may lead to postoperative abdominal discomfort. After finishing the repair, an intercostal tube was inserted, and the incision was closed in layers. An intercostal nerve block with xylocaine was used for analgesia. All patients were extubated directly in the operating room.

\section{Statistical analysis:}

Statistical analysis was performed with SPSS (SPSS version 22) the result expressed as mean \pm SD for patients' characteristics, the level of the everted diaphragm in $\mathrm{cm}$, and spirometry. Univariety and multi variety analysis were compared, Pearson's correlation was used for correlation analysis, a value of $p$ $<0.05$ was considered statistically significant.

\section{Results:}

There were 40 patients underwent SDP. 30 (75\%) males and $10(25 \%)$ females (the mean age was $45 \pm 24$ years), other patients' characteristics were analyzed (Table 1). Seven patients were excluded due to incomplete data. 31 patients (78\%) were presented with dyspnea, 6 patients were suffering from deterioration of their sports activities (15\%), 3 patients were diagnosed accidentally using CXR (7\%). CXR showed elevated diaphragm on one side in 39 patients (98\%) and on both sides in one patient (2\%). The diagnosis of the elevated diaphragm was confirmed in all patients for $<3$ years in 12 patients $(30 \%)$, between a 3-1 year in 14 patients (35\%) and < one year in 14 (35\%). 34 cases were idiopathic, and 5 cases were post-cardiac surgery and one patient after cryoablation. The degree of eventration was $>8 \mathrm{~cm}$ 
in $15 \%(n=6), 8-5 \mathrm{~cm}$ in $75 \%(n=30)$, and $<5 \mathrm{~cm}$ in $10 \%(n=4)$. The US showed no diaphragmatic movement on the affected side in all patients but paradox movement in $85 \%(n=34)$. Lung functions showed restricted values in $80 \%(n=32)$, combined restricted and obstructed values in $12.5 \%(n=5)$ and incomplete work in $7.5 \%(n=3)$, other patients characteristics are summarized in Table 1. SDP was done more on the left side $57.5 \%(n=23)$. Mean length of surgery was $48.8 \pm 19.7$ (range: $30-70)$ min. Postoperative overall morbidity was $7.5 \%$, with no mortality. One patient suffered from pneumonia treated with antibiotics and oxygen. Two cases complained from chronic post-thoracotomy pain relieved by analgesics. Follow up at one 6 months and two years postoperatively showed a significant increase in FEV1 and FVC $(P<0.001$ and $P<0.01)$ respectively (Fig. 4 a,b). Arterial blood gas analysis showed a significant drop in the $\mathrm{CO} 2$ values under exercise compared with preoperative values $(P<0.001) .6$ minutes' walk distance was significantly improved in $95 \%(n=38)(P<0.01)$. Additionally, there was a significant postoperative decrease in the difference in height between plicated and the normal diaphragm on a CXR ( $P=0.034)$. No paradoxical movement of the diaphragm was noticed postoperatively $(P<0.001)$. Likewise, there was a significant postoperative improvement in dyspnea $(P<0.001)$. There was a significant correlation between postoperative FEV1 and the hight of the copula on CXR and DUS on inspiration and expiration ( $\mathrm{P}<0.001$ and $\mathrm{P}<0.005$ respectively). Also, was observed at 6 months and 2 years postoperative follow up (Fig. $5 \mathrm{a}, \mathrm{b}$ ). The mean hospital stay was 5 days. The mean duration of chest tube drainage was 3 days. No recurrence was detected throughout a mean follow-up of 36 months.

\section{Discussion:}

Although DP is a known condition which is not uncommon in daily practice, still many general practitioners and pneumologists are not aware of its surgical management. Many patients are suffering under dyspnea over many years even though they are under complex conservative treatment. It may be congenital, neurogenic (central or peripheral), atrophic, aplastic, hypoplastic, iatrogenic or infectious. Orthopnoea and Dyspnea are the main indications for SDP [2]. Although the diagnosis of DP is easy to achieve using CXR, still, a large number of patients are suffering from severe dyspnea and deterioration of their daily activities with bad quality of life and offered no treatment apart from inhalation therapy.

Surgical plication of the diaphragm is a well-known procedure, but it is still a seldom done one among general surgeons. Thoracotomy is known as one of the most painful incisions, which needs thoracic surgery experience, and it is not always easy to manage among general surgeons. Therefore many patients are denied surgical treatment. The current study, included 40 cases with UDP that was more common on the left side (57\%), dyspnea was the most common symptom, preoperative FEV1 and FVC were closely correlated with the degree of eventration of the diaphragm as evaluated by CXR and DUS, also there was a significant correlation between postoperative FEV1 and degree of correction of diaphragmatic eventration as evaluated by CXR and DUS, at six months and two years postoperative.

During the plication, it is important to be cautious and to pull the diaphragm up to avoid injury of any abdominal organs. The diaphragm became tough and firm and returned to its normal position. 
Furthermore; it is important not to do overcorrection, as it might have more pressure on the other side of the diaphragm or may lead to postoperative abdominal discomfort.

In a study that included 17 patients conducted by Graham et al, it was found that SDP results in significant recovery in respiratory distress and respiratory function test [14]. Similarly, Higgs et al followed 19 patients for 10 years and reported that SDP is a suitable choice to improve respiratory function values, dyspnea score and patient satisfaction [15]. Freeman et al reported a recovery in spirometry values in 41 case series at 6 months and over long-term follow-up [16]. I a study by Ribet he found a 15\% increase in FEV1 and a 20\% increase in vital capacity following plication [17]. Also; Calvinho et al, described the long-term follow-up of 20 patients with a maximum of up to 17 years, most of the patients had an important impairment of their daily activities preoperatively and all except three improved their physical status [18]. Most of the above-mentioned studies were done on children or patients on respiratory support. Our study with a bigger number of patients showed similar results with improvement of the FEV1 of $20 \%$ and FVC of $15 \%$ with long-lasting effects in adult patients. On the other hand, Özkan et al found that there is an improvement in postoperative FEV1 at one month, however, neither a statistically nor a clinically significant increase was observed in long-term results [19]. Our results show significant improvement in respiratory functions later on six months and two years.

Chest ultrasonography is more helpful, in the evaluation of cases of diaphragmatic eventration than CXR, at start it is helpful in diagnosis which can be confirmed by paradoxical movement of highly positioned diaphragmatic copula, also postoperative sonography evaluation is helpful for assessment of degree of correction of eventration and to detect paradoxical movement if any [20].

\section{Conclusion:}

We concluded that adult patients with chronic dyspnea due to UDP significantly benefit from SDP as it is safe and correlated with a significant improvement in patient functional status which is closely correlated with a radiological and sonographic assessment.

\section{Abbreviations}

CXR: Chest X-ray

DP: Diaphragmatic paralysis

DUS: Diaphragm ultrasonography

FEV1: Forced expiratory volume 1

FVC: Forced vital capacity

SDP: Surgical diaphragmatic plication 


\section{Declarations}

\section{Acknowledgments:}

To the patients who included in the study and came back to our hospital at pre-determined regular visits for follow up.

Availability of data and materials:

Not applicable.

Consent for Publication:

Not applicable.

\section{Funding:}

This research did not receive a grant from any funding agency in the public or commercial sectors.

\section{Authors' contributions:}

Mohamed Abdel Bary and Alaa Rashad contributed substantially to the study design, data analysis and interpretation, and the writing of the manuscript.

Hamed Elgendy contributed substantially to data editing and statistical analysis.

Morris Beshaycontributed substantially to figures design and revision of the manuscript.

Ethics approval and consent to participate:

The study conforms to the ethical standards of the Helsinki Declaration and approval was obtained from the institutional ethics committee of South Valley University Hospital.

\section{Competing interests;}

The authors have declared that no competing interest. 


\section{References}

[1] Mouroux J, Venissac N, Leo F, Alifano M, Guillot F. Surgical treatment of diaphragmatic eventration using video-assisted thoracic surgery: a prospective study. Ann Thorac Surg. 2005;79:308-12.

[2] Gazala S, Hunt I, Bédard EL. Diaphragmatic plication offers functional improvement in dyspnea and better pulmonary function with low morbidity. Interact Cardiovasc Thorac Surg. 2012;15(3):505-8.

[3] Gatzinsky P, Lepore V. Surgical treatment of a large eventration of the left diaphragm. Eur J Cardiothorac Surg. 1993;7:271-4.

[4] Nathani N, lles PB. Acute respiratory failure with an abnormal chest radiograph. Respiration 2005;72:205-9.

[5] Onders PR. Physiology of the Diaphragm and Surgical Approaches to the Paralyzed Diaphragm. In: LoCicero III J, Feins RH, Colson YL, Rocco G, editors. Shields' General Thoracic Surgery. 8th ed. Wolters Kluwer; 2019:1383-1398.

[6] Elshafie G, Acosta J, Aliverti A, et al. Chest wall mechanics before and after diaphragm plication. J Cardiothorac Surg. 2016;11:25.

[7] Gruber PJ. Diaphragm plication: When and why to do it. J Thorac Cardiovasc Surg. 2017;154(5):171213.

[8] Freeman RK, Wozniak TC, Fitzgerald EB. Functional and physiologic results of video-assisted thoracoscopic diaphragm plication in adult patients with unilateral diaphragm paralysis. Ann Thorac Surg. 2006;81:1853-57.

[9] Groth SS, Andrade RS. Diaphragm plication for eventration or paralysis: a review of the literature. Ann Thorac Surg. 2010;89:2146-50.

[10] Versteegh MI, Braun J, Voigt PG, et al. Diaphragm plication in adult patients with diaphragm paralysis leads to long-term improvement of pulmonary function and level of dyspnea. Eur $\mathrm{J}$ Cardiothorac Surg. 2007;32(3):449-56.

[11] Demos DS, Berry MF, Backhus LM, Shrager JB. Video-assisted thoracoscopic diaphragm plication using a running suture technique is durable and effective. J Thorac Cardiovasc Surg. 2017;153(5):1182- 
88.

[12] Celik S, Celik M, Aydemir B, Tunckaya C, Okay T, Dogusoy I. Long-term results of diaphragmatic plication in adults with unilateral diaphragm paralysis. J Cardiothorac Surg. 2010;5:111.

[13] Ueki J, De Bruin PF, Pride NB. In vivo assessment of diaphragm contraction by ultrasound in normal subjects. Thorax. 1995;50(11):1157-61.

[14] Graham DR, Kaplan D, Evans CC, Hind CR, Donnelly RJ. Diaphragmatic plication for unilateral diaphragmatic paralysis: a 10-year experience. Ann Thorac Surg. 1990;49(2):248-51; discussion 252.

[15] Higgs SM, Hussain A, Jackson M, Donnelly RJ, Berrisford RG. Long term results of diaphragmatic plication for unilateral diaphragm paralysis. Eur J Cardiothorac Surg. 2002;21(2):294-7.

[16] Freeman RK, Van Woerkom J, Vyverberg A, Ascioti AJ. Long-term follow-up of the functional and physiologic results of diaphragm plication in adults with unilateral diaphragm paralysis. Ann Thorac Surg. 2009;88(4):1112-7.

[17] Ribet M. Diaphragmatic plication. Ann Thorac Surg. 1990;50:855-56.

[18] Calvinho P, Bastos C, Bernardo JE, Eugénio L, Antunes MJ. Diaphragmatic eventration: long-term follow-up and results of open-chest plicature. Eur J Cardiothorac Surg. 2009;36(5):883-7.

[19] Özkan S, Yazici Ü, Aydin E, Karaoğlanoğlu N. Is surgical plication necessary in diaphragm eventration? Asian J Surg. 2016;39(2):59-65.

[20] Farghaly S, Hasan AA. Diaphragm ultrasound as a new method to predict extubation outcome in mechanically ventilated patients. Aust Crit Care. 2017;30(1):37-43.

\section{Table 1}

Table 1: Demographic data of study cases 


\begin{tabular}{|c|c|c|}
\hline Variable & & \\
\hline Age years (mean $\pm \mathrm{SD})$ & & $45 \pm 14$ \\
\hline Sex & & \\
\hline Male / Female & & $30 / 10$ \\
\hline Lateralization & & \\
\hline Left/ right & & $23 / 17$ \\
\hline Spirometry (\% predicted) & & \\
\hline FVC $($ mean $\pm S D)$ & & $60 \pm 14$ \\
\hline FEV1 (mean \pm SD) & & $68 \pm 13$ \\
\hline FEV1/FVC (mean \pm SD) & & $81 \pm 11$ \\
\hline CXR height of diaphragm (cm) & & $8 \pm 4$ \\
\hline DUS height of diaphragm (cm) & & $8 \pm 5$ \\
\hline Dyspnea (MRC) & & \\
\hline & 2 & $3(7.5 \%)$ \\
\hline & 3 & $28(70 \%)$ \\
\hline & 4 & $9(22.5 \%)$ \\
\hline
\end{tabular}

\section{Figures}


Fig. 1

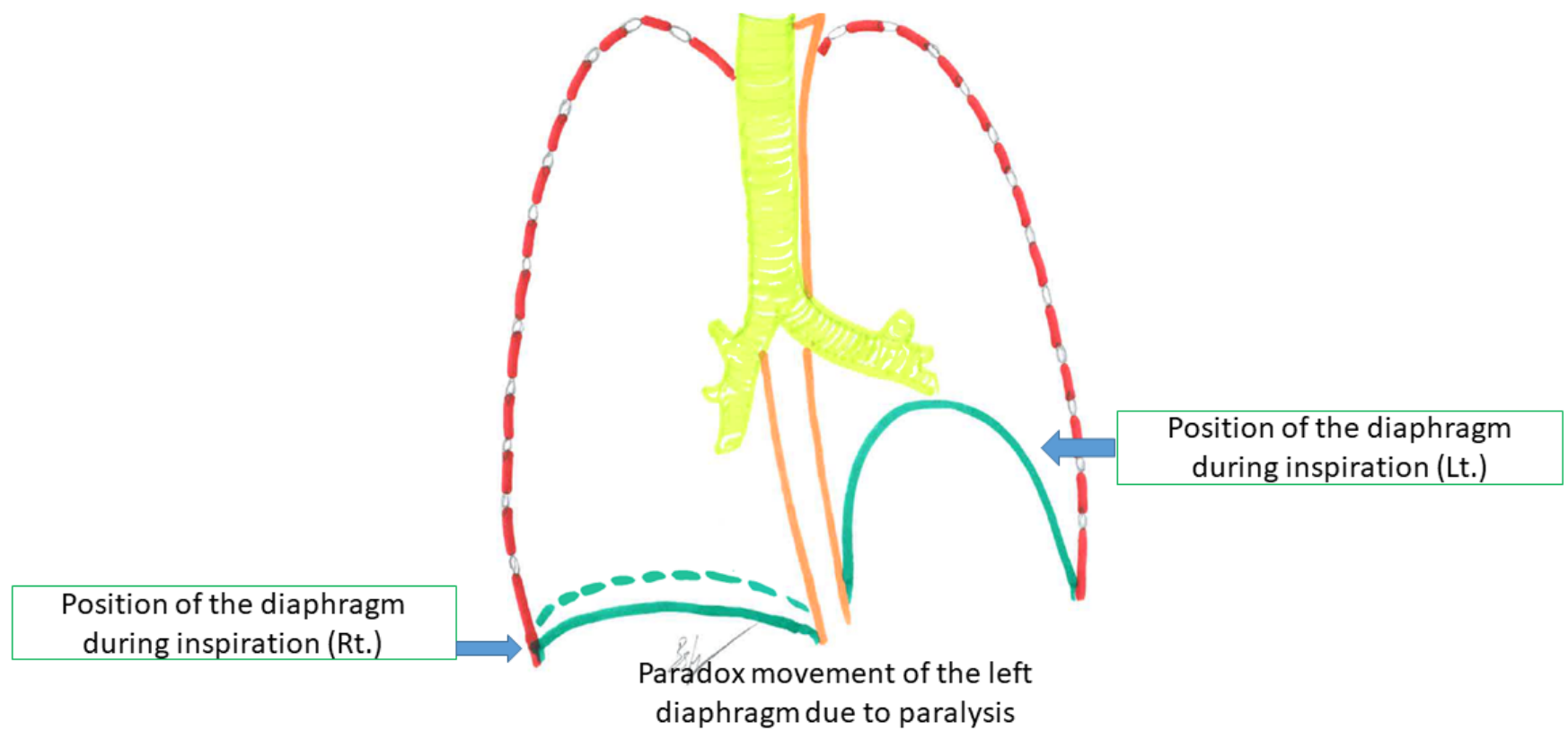

Figure 1

Paradox movement of the left diaphragm due to paralysis.

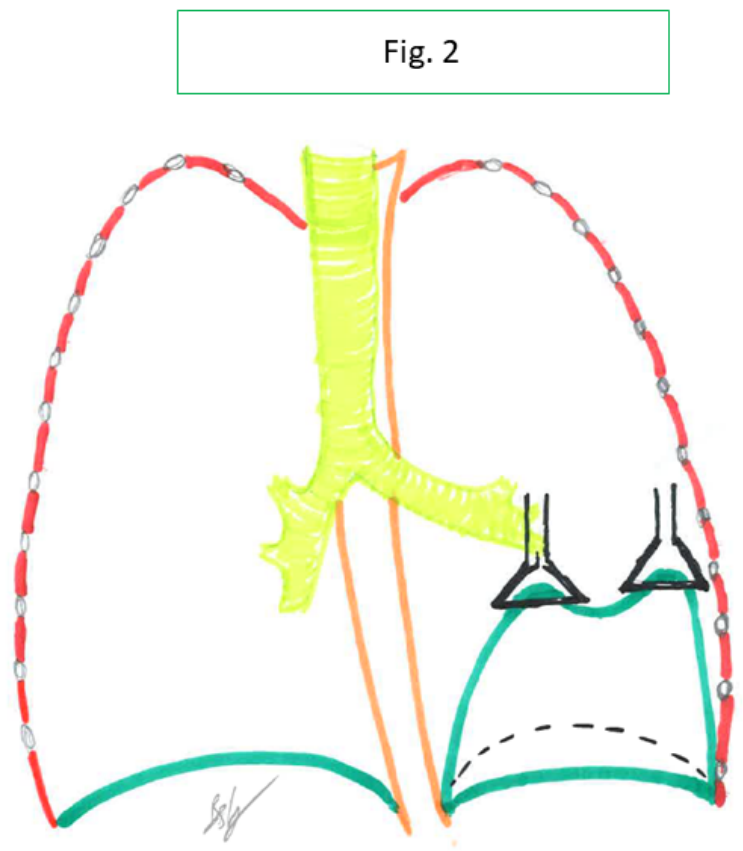

First step of diaphragmatic plication: making a fold of the paralysed diaphragm 
Figure 2

First step of diaphragmatic plication: making a fold of the paralysed diaphragm.

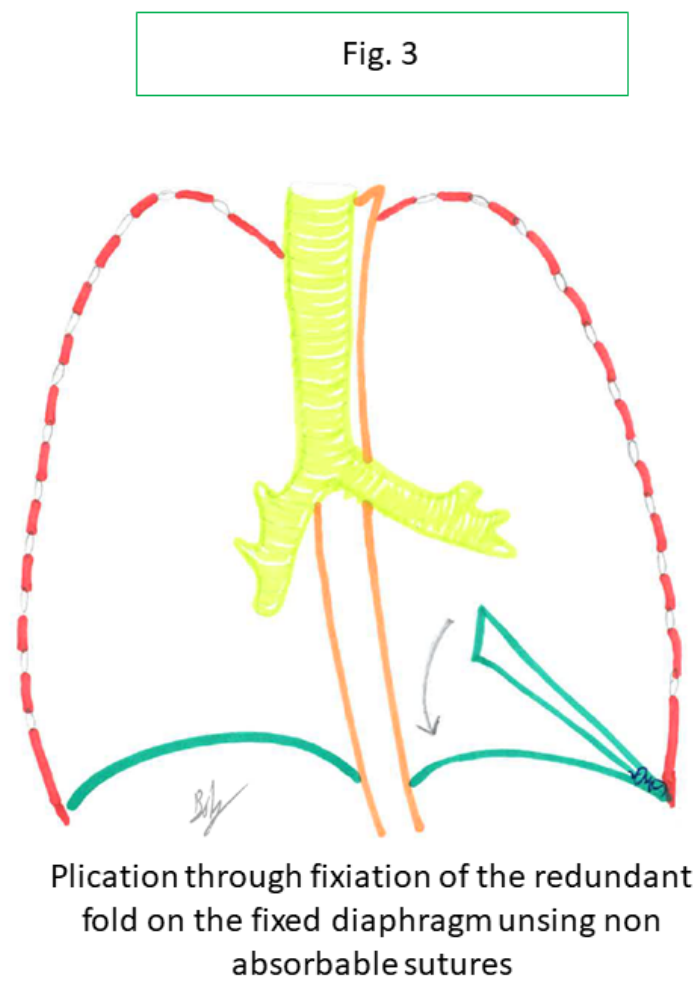

\section{Figure 3}

Plication through fixation of the redundant fold on the fixed diaphragm using non-absorbable sutures.
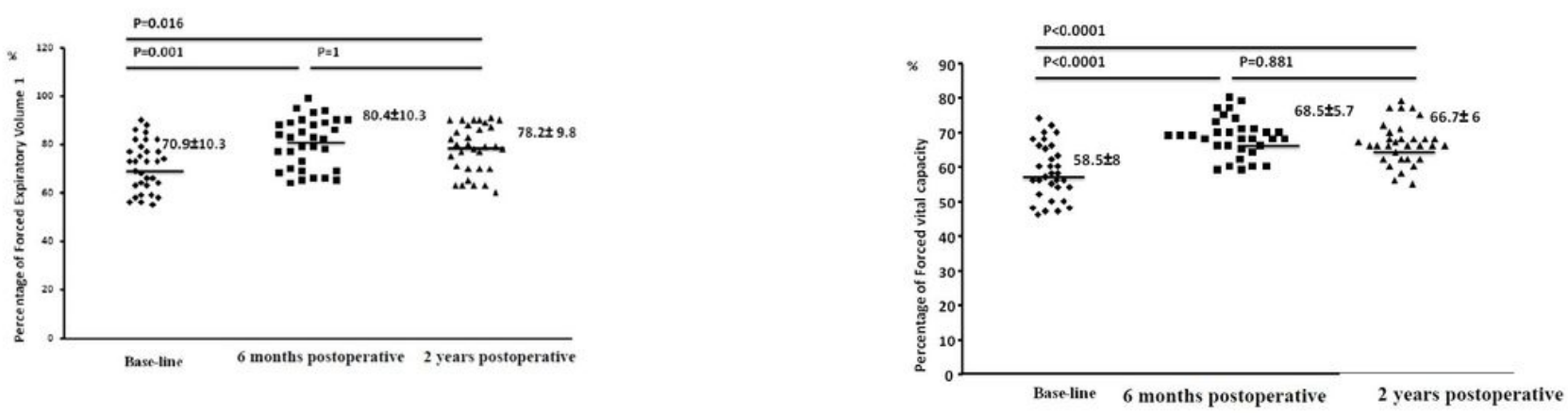

\section{Figure 4}

a: Correlation of forced expiratory volume 1 (FEV1) at baseline, 6 months and 2 years postoperatively. b: Correlation of forced vital capacity (FVC) at baseline, 6 months and 2 years postoperatively. 

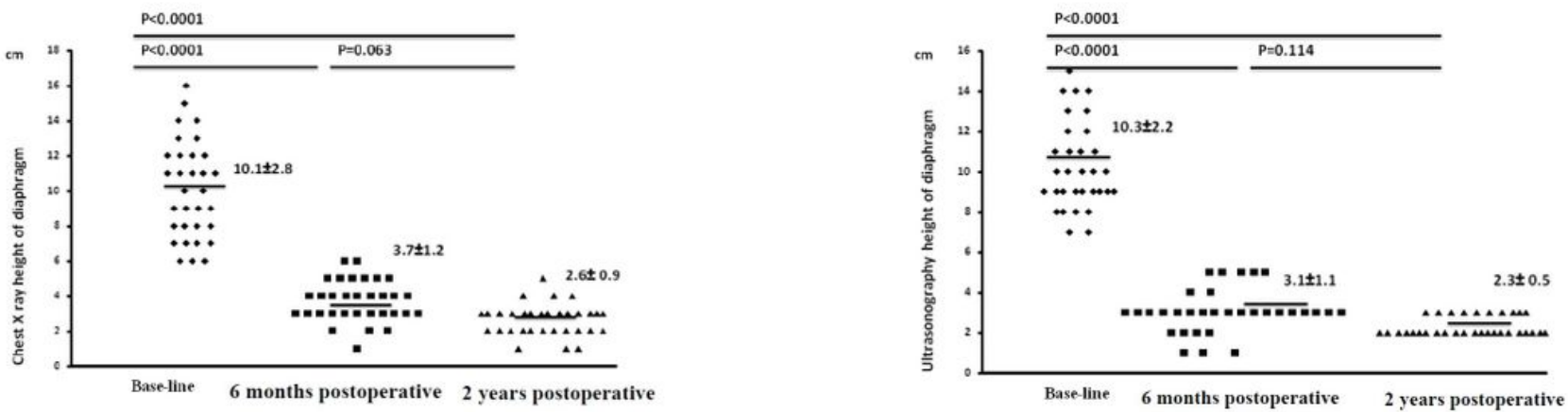

\section{Figure 5}

a: Correlation of the height of the diaphragm $(\mathrm{cm})$ by chest X-ray (CXR) at baseline, 6 months and 2 years postoperatively. $\mathrm{b}$ : Correlation of the height of the diaphragm $(\mathrm{cm})$ by diaphragm ultrasonography (DUS) at baseline, 6 months and 2 years postoperatively. 